\title{
Laplacian-Gaussian Sub-Correlation Analysis for Scale Space Imaging
}

\author{
Kohji Kamejima \\ Faculty of Information Science and Technology, Osaka Institute of Technology \\ 1-79-1 Kitayama, Hirakata 573-0196 JAPAN \\ e-mail: kamejima@is.oit.ac.jp
}

\begin{abstract}
Two universal imaging rules, self-similarity and perspective projection, are combined for detecting almost horizontal space in complex scene. By extracting scale shift due to perspective projection through Laplacian-Gaussian image analysis, the expansion of maneuvering area is estimated in terms of the invariant measure associated with a fractal attractor. Estimated maneuvering area is shown to be visualized on the scene image without explicit geometry of the roadway. The representation scheme was verified through experimental studies.
\end{abstract}

Keywords: $\quad$ Scene Analysis; Perspective Projection; Self-Similarity; Maneuvering Affordance

\section{Introductory Remarks}

Visual perception organizes randomly distributed image features into cues to the recognition of encountered scene. As an $a s$-is part of real world, natural object itself maintains consistency within the scene independent of observer specific context. This implies the existence of universal rules governing the intrinsically invariant structure of the scene. By computing variations of contexts, a definite set of universal rules, if exists, expands all possible aspects of real world as logically decidable scenes. Noting the synchronizability of symbolic computation and image analysis in perception processes [11], hence, essential capability of the vision system as 'real world intelligence', either it has evolved naturally or designed artificially, is the re-cognition of computable scenes: mechanical application of the universal rules as the common belief on real world to imitate the incubation process of preestablished goal within observed image of the scene. Such intentionally nondeterministic image analysis takes a crucial role in various implementation of end user programming [7], knowledge reuse [12], the open logic machine [11], driver's associate [5] and explorative education [3], e.g.

As computational basis of the recognition process, the universal rule underlying the scene should be of linguistic structure enough for the vision system to manipulate referential objects computationally. The representation of the universal rules, simultaneously, should be of sufficient diversity for the vision system to be predictively adaptive to the scene. The substantiation of universal rules with nondeterministic representation has been one of central problems in natural and/or artificial perception. Following ecological optics [6], in fact, stable 3D structure of naturally complex scene is scattered in the perspective of random texture on the earth. Various mental reactions to mathematically well

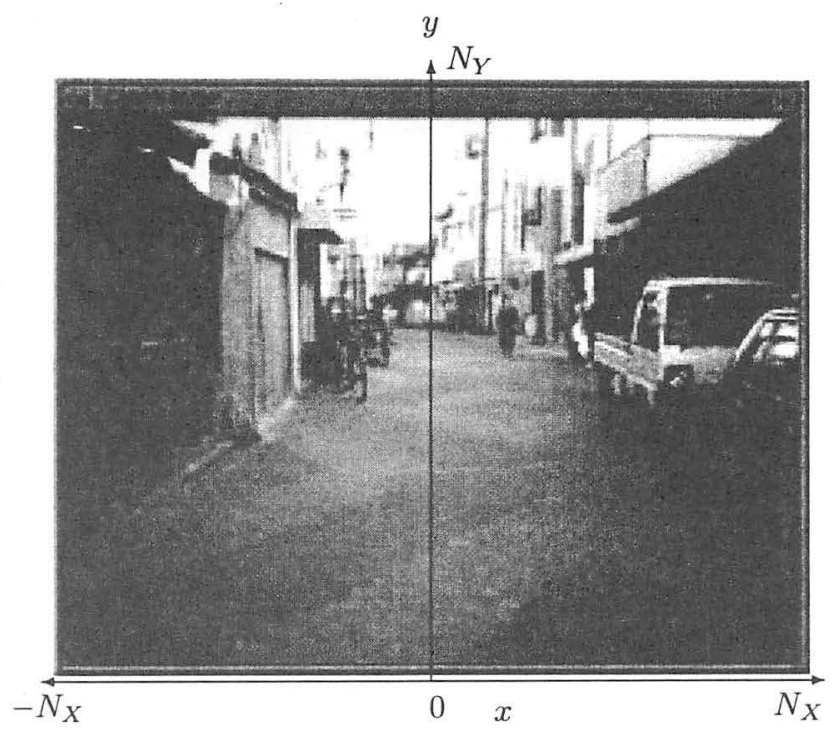

Figure 1: Scene Imiage with Distribution $f(x, y)$

arranged visibles can be explained as the responses of simple mechanisms [2], [4], [14]. In engineering applications, 2D distributed representation of perspective was demonstrated to take a crucial role in visual guidance systems [13].

Consider an example of complex scene as shown in Fig. 1 where the problem is to decide the space for possible maneuvering based on a system of universal rules. In this situation, observed image is represented as a mathematical entity: the distribution $f$ on a fixed image plane $\Omega \subset R^{2}$ consisting of pixels $\omega=(x, y) \in \Omega$, $x \in\left[-N_{X}, N_{X}\right], y \in\left[0, N_{Y}\right]$. The goal is to describe the scene in terms of referent objects including roadway, obstacles, landmark sign and pedestrians, e.g. Without serious loss of generality, the space of referent objects 


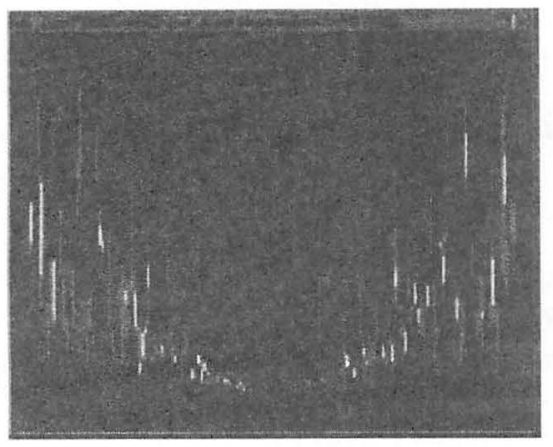

Figure 2: Power Spectrum of Line Image: $\Delta f(\cdot, 0)$

Power spectrum of the brightness distribution is computed on the bottom line of the image plane $(x, 0) \in \Omega$, $x \in\left[-N_{x}, N_{x}\right]$. To enhance random component which is expected to be distributed uniformly on the roadway, the distribution of twice differential image $\Delta f(x, y)$, $x \in\left[-N_{x}, N_{x}\right], y=0$ is used.

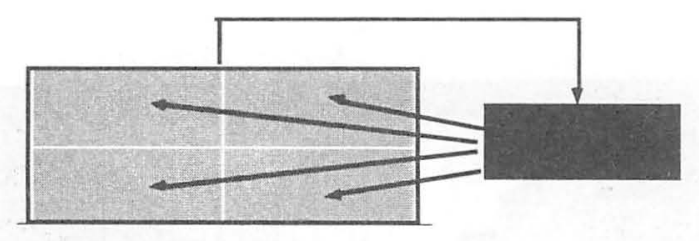

Figure 3: Self-Similarity Process $\nu$

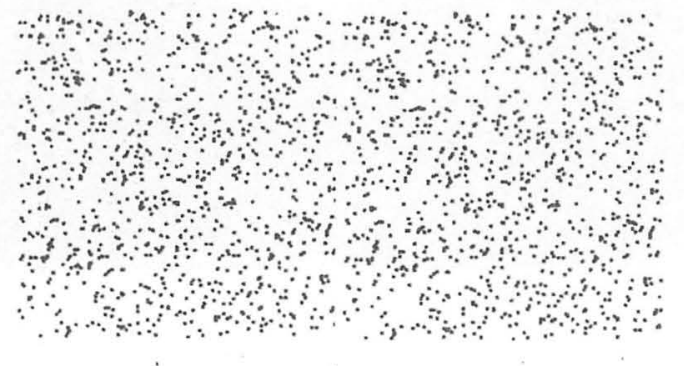

Figure 4: Associated Fractal Attractor $\Xi$

can be identified with the Borel field $\mathcal{F}[\Omega]$ : the totality of subsets of $\Omega$. In this paper, we consider dynamic organization of random image features under propositionally specified vehicle's intention. For this purpose, in what follows, two types of universal rules, i.e., selfsimilarity and perspective projection, are combined for organizing image features into a cognitive entity, called maneuvering affordance [9].

\section{Existence of Perceptible Complexity}

The surface of real object is engraved with complex texture through intentional ornamentation and natu- ral weathering processes. The complexity of the roadway in Fig. 1 is evaluated in Fig. 2 where the power spectrum at the bottom of the scene image is indicated. To extract the support of the complexity in the image, the Fourier transform was applied to the twice spatial derivative of the brightness distribution $f(x, 0)$. As shown in Fig. 2, we can confine the roadway area into the support of random distribution modulated by artificial ornamentation. Recent advances in fractal mathematics provides a simple dynamics to generate random distribution. For instance, the iteration of reduction-collage process as shown in Fig. 3 yields random distribution filling a rectangle as illustrated in Fig. 4. Exactly, the rectangle is demonstrated to be identified with a fractal attractor $\Xi$ satisfying the following self-similarity

$$
\Xi=\bigcup_{\mu_{i} \in \nu} \mu_{i}(\Xi)
$$

where $\mu_{i}: \Omega \mapsto \Omega$ is reduced affine mapping specifying the iterated collage process. Noting uncertainty in (1), let the attractor $\Xi$ be approximated successively by the following nondeterministic dynamical system

$$
\xi_{t+1}=\mu_{i}\left(\xi_{t}\right),
$$

where mapping $\mu_{i} \in \nu$ is randomly selected at each iteration step with probability $p_{i}, 0 \leq p_{i} \leq 1$, such that

$$
\sum_{\mu_{i} \in \nu} p_{i}=1
$$

Despite intrinsic unpredictability, the dynamical system (2) leaves the vestiges as an image strictly obeys the self-similarity:

Proposition 1 (Measurability) [1] There exists an measure $\chi_{\Xi}^{\mathfrak{p}}$ invariant with respect to the following Markov operation associated with imaging dynamics (2):

$$
\chi_{\Xi}^{\mathfrak{p}}(\cdot)=\sum_{\mu_{i} \in \hat{\nu}} p_{i} \chi_{\Xi}^{\mathfrak{p}}\left[\mu_{i}^{-1}(\cdot)\right], \quad(\cdot) \in \mathcal{F}[\Omega] .
$$

As the visualization of the attractor in the following sense

$$
\operatorname{supp} \chi_{\Xi}^{p}=\Xi
$$

the invariant measure $\chi_{\Xi}^{p}$ provides crucial cue to identify program set $\nu$ based on resulted imagery. The combination of dynamic randomness (2) with nondeterministic constraint (1), thus, yields the following stochastic evaluation within the framework of entropy maximization:

Proposition 2 (Detectability) [8] The probability for capturing unknown attractor $\Xi$ is represented by the solution $\varphi(\omega \mid \nu)$ to the following equation:

$$
\frac{1}{2} \Delta \varphi(\omega \mid \nu)+\rho\left[\chi_{\Xi}^{p}-\varphi(\omega \mid \nu)\right]=0
$$




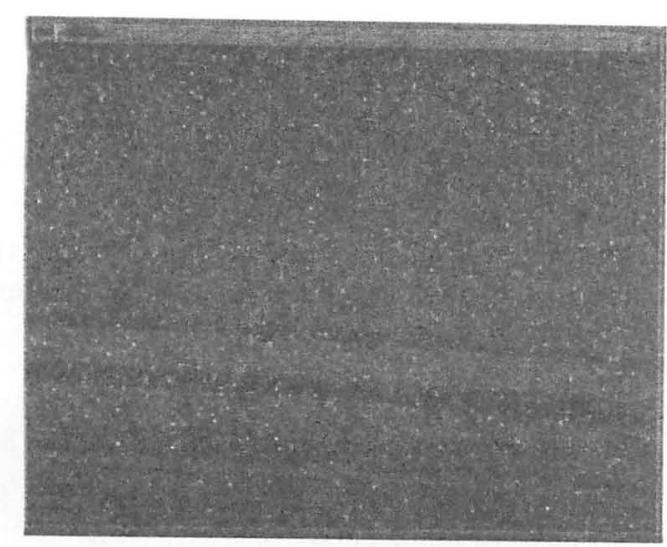

Figure 5: Noisy Strip $\chi_{\Xi}^{\mathfrak{p}}$

where $\rho=\log \|\nu\|$ is the complexity parameter specified in terms of the size of mapping set $\|\nu\|$.

To generate the capturing probability $\varphi(\omega \mid \nu)$, it is sufficient to estimate the complexity index of imaging process $\|\nu\|$. Furthermore, we can select most probable points of not-yet-identified attractor $\Xi$ through local analysis of multiscale imagery:

$$
\tilde{\Theta}=\left\{\tilde{\theta} \in \Lambda \mid \nabla \varphi=0, \operatorname{det}\left[\nabla \nabla^{T} \varphi\right]>0, \Delta \varphi<0\right\} .
$$

The stochastic features $\tilde{\Theta}$ has been proved to satisfy the following computational consistency:

Proposition 3 (Finite Computability) [8] There exists an subset $\Theta \subset \tilde{\Theta}$ invariant with respect to $\nu$, i.e.,

$$
\Theta=\left\{\theta \in \tilde{\Theta} \mid \exists \mu_{i} \in \nu: \mu_{i}^{-1}(\theta) \in \Theta\right\} .
$$

The combination of propositions 2 and 3 provides computational basis for self-similarity coding of complex random patterns. The coding scheme has been demonstrated to extract the self-similarity in noise dominant observation. Thus, we have the implementation of the first universal rule, the self-similarity, as Eqs. (4) and (6). For instance, by substituting almost noise pattern (Fig. 5) for $\chi_{\Xi}^{\mathfrak{p}}$, the diffusion mechanism (4) filters the outline of the self-similarity region (Fig. 6) to verify the finite invariance as shown in Fig. 7. Hence, fractal modeling of the roadway area results in the transformation of the brightness distribution $f(\omega)$ into invariant measure $\chi^{p}$.

\section{Intentional Imaging of Complex Perceptant}

On the earth, the direction of the gravity force induces a natural $3 \mathrm{D}$ interpretation rule in image plane. Noticing the existence of perceptible complexity, consider 3D coordinate system as illustrated in Fig. 8. The complex perceptant $\chi_{\Xi}^{p}$ is skewed through the coordinate

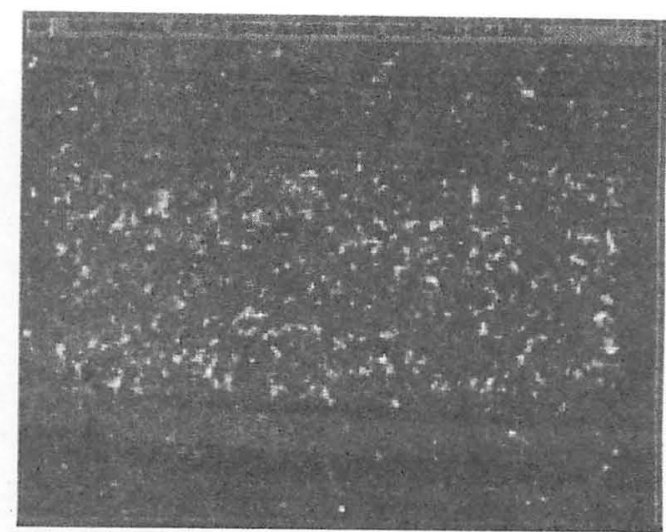

Figure 6: Capturing Probability $\varphi(\omega \mid \nu)$

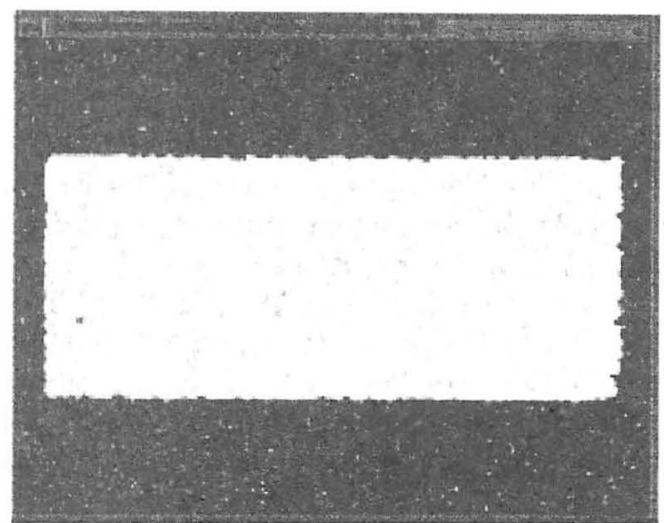

Figure 7: Restored Fractal Attractor

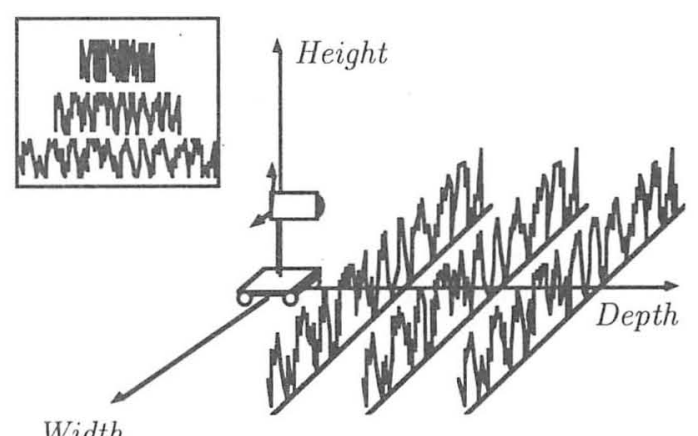

Figure 8: Intentional Coordinate System

system to evoke the distribution $f$ in association with the intention of motion. Evoked distribution $f$ has the representation

$$
\chi_{X}^{\mathfrak{F}}\left(\omega_{x}, y\right)=\int_{\mathbf{X}} f(x, y) e^{-j \omega_{x} x} d x,
$$




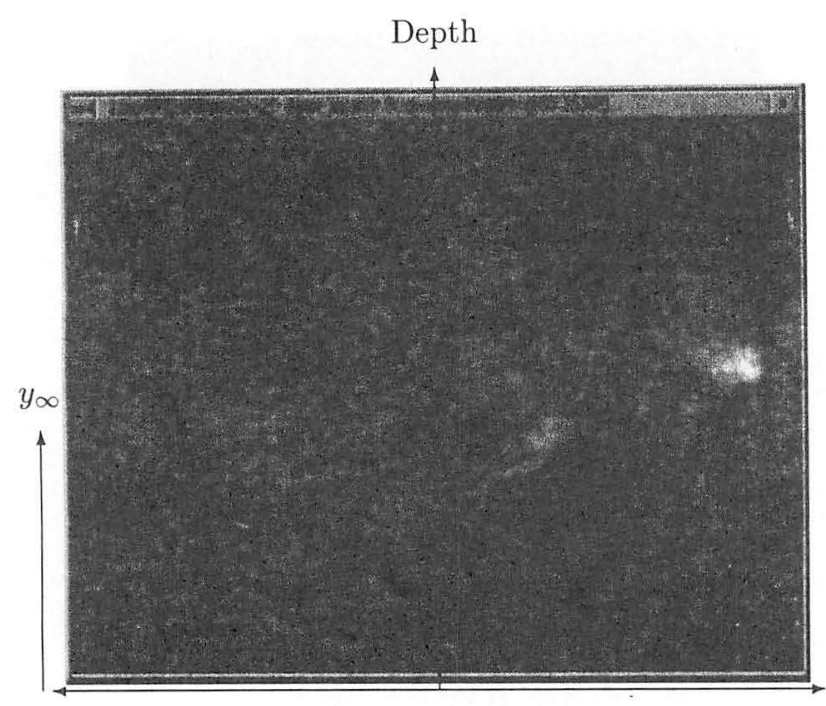

Frequency

Figure 9: Directional Fourier Transform of $\Delta f$

where $\chi_{X}^{\mathfrak{F}}\left(\omega_{x}, y\right)$ denotes the directional Fourier image with respect to the horizontal frequency $\omega_{x}$ and vertical position $y$. The directional Fourier image $\chi_{X}^{\mathfrak{F}}\left(\omega_{x}, y\right)$ associated with the scene (Fig. 1), is shown in Fig. 9. This figure implies that the depth of nearly horizontal plane is detectable in terms of the interval $\left[0, y_{\infty}\right]$ on which power spectrum of line images $\left\{f((\cdot), y), 0 \leq y \leq y_{\infty}\right\}$ follows the similarity representation in frequency domain.

\section{2D Laplacian-Gaussian Sub-Correlation}

The existence of the vanishing depth $y_{\infty}$ implies the detectability of the support for the maneuvering without explicit information of the roadway. Let $\sigma(\omega)$ be dominant scale of the distribution $f(\omega)$ in $\Omega$ : Noticing that the pixel in the directional Fourier image represents the pair $(1 / \sigma, y)$, we can estimate the skewed image of the invariant measure $\chi_{\Xi}^{\mathfrak{p}}$ as the set of pixels $\hat{\chi} \Xi \in \mathcal{F}[\Omega]$ in which scale components of the distribution $f(\omega)$ are regulated by the similarity induced by the intentional coordinate system.

Consider bottom up generation of the estimate $\hat{\chi} \equiv$. Noting the locating process (2), the measure $\chi_{\Xi}^{p}$ can be identified with the aggregation of point images $\delta_{\xi}$ located at $\xi \in \Xi \subset \Omega$. Through the association

$$
\Xi \ni \xi \Longleftrightarrow \delta_{\xi} \in \chi_{\Xi}^{p},
$$

each point image $\delta_{\xi}$ is transformed to smooth gray level distribution through the Gaussian point spread function given by

$$
g_{\sigma}(\omega \mid \xi)=\frac{1}{2 \pi \sigma^{2}} \exp \left[-\frac{|\omega-\xi|^{2}}{2 \sigma^{2}}\right],
$$

where $\sigma$ denotes the scale parameter. By selecting the parameter $\sigma$, conversely, specific scale component of the measure $\delta_{\xi}$ can be filtered. Since

$$
\sigma^{2}\left|\frac{1}{2} \Delta g_{\sigma}(\tilde{\theta} \mid \xi)\right|=g_{\sigma}(\tilde{\theta} \mid \xi)
$$

at local maximum point $\tilde{\theta}$ of $g_{\sigma}$, we can estimate the scale parameter $\sigma$ based on observables $\Delta g_{\sigma}(\tilde{\theta} \mid \xi)$ and $g_{\sigma}(\tilde{\theta} \mid \xi)$ without explicit information $\xi$.

Generally, consider the following decomposition

$$
f_{\sigma}(\omega) \sim g_{\sigma} * \chi_{\Xi}^{\mathfrak{p}}(\omega)+b(\omega),
$$

where $b(\omega)$ denotes nonnegative bias satisfying

$$
|\Delta b| \ll\left|\Delta g_{\sigma}\right| .
$$

Noting biased correlation $\sigma^{2}\left|\frac{1}{2} \Delta f(\tilde{\theta})\right| \sim f(\tilde{\theta})-b(\tilde{\theta})$, we have

Proposition 4 (Sub-Correlation) The upper bound for scale parameter $\sigma$ at $\tilde{\theta}$ can be estimated by

$$
\sigma^{2}(\tilde{\theta}) \leq \frac{f(\tilde{\theta})}{\left|\frac{1}{2} \Delta f(\tilde{\theta})\right|},
$$

at each local maximum point $\tilde{\theta}$ of the imagery $f(\omega)$, $\omega \in \Omega$.

The weak constraint (9) can be extracted via local analysis based on $f$ and $\Delta f$. This implies we have noisy observation for the distribution of $\sigma$-scale image through $f-\Delta f$ association.

Consider the following representation at $\omega=(x, y)$ :

$$
f_{\sigma}(\omega)=\left|f_{\sigma}\right| \exp \left[2 \pi j \frac{x+y}{\sigma}\right] .
$$

Noticing

$$
\begin{aligned}
\frac{f_{\sigma+\epsilon}(\omega)}{f_{\sigma}(\omega)} & =\mathfrak{R e}\left[\exp \left[2 \pi j \frac{\epsilon(x+y)}{\sigma(\sigma+\epsilon)}\right]\right] \\
& \sim \frac{1}{2 \pi} \exp \left[-\frac{|\epsilon|^{2}}{2}\right] d \omega
\end{aligned}
$$

scale deviation $\epsilon$ can be indexed in terms of the following normal distribution

$$
n_{\sigma}(\epsilon)=\frac{1}{2 \pi} \exp \left[-\frac{\epsilon^{2}}{2}\right] .
$$

Hence we have the measure indicating the probability for detecting $\sigma$-scale component. The scale space feature $n_{\sigma}(\epsilon)$ generated by the scene image is shown in Fig. 10 where the vanishing point $y_{\infty}$ is guessed at $N_{y}$ and the scale parameter is shifted as follows

$$
\sigma(y)=\frac{\sigma_{0}}{y_{\infty}}\left(y_{\infty}-y\right) .
$$




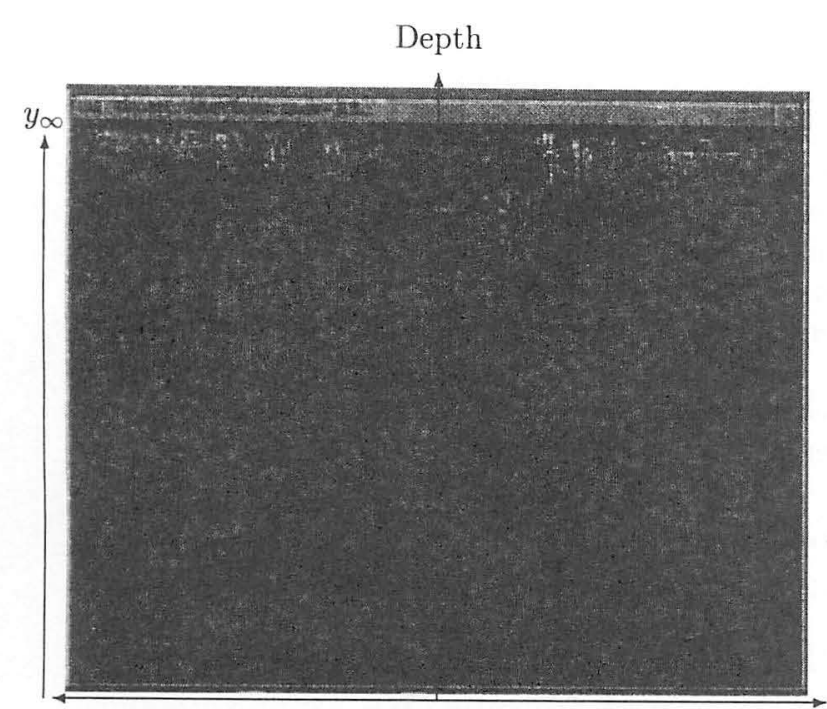

Expansion

Figure 10: Multi-Scale Image $n_{\sigma}(\epsilon)$

In eq. (12), $\sigma_{0}$ is adjusted to the dominant scale of $\Delta f\left(x, y_{0}\right)$. By evaluating

$$
\chi_{\Xi}^{\mathfrak{p}} \sim \hat{\chi} \Xi=n_{\sigma(y)} f,
$$

in (4), we have the capturing probability for underlying fractal attractor as the diffusion field generated by the excitation $\hat{\chi} \Xi$.

\section{Fractal Model Matching}

Perspective projection induces the proportionality (12) to yield skewed version of the invariant measure $\hat{\chi} \Xi$. Let the support of the proportionality be visualized in terms of a fractal attractor with fixed points

$$
\Omega_{\nu}^{f}=\left\{\omega_{\mu_{i}}^{f}, \mu_{i} \in \nu\right\}
$$

as illustrated in Fig. 11 [10] and consider the estimation of the generic model $\Omega_{\nu}^{f}$ based on the following one parameter family of linear distributions

$$
\begin{aligned}
\tilde{\Theta}_{x} & =\left\{\tilde{\Theta}_{x}(y) \mid 0 \leq y \leq y_{\infty}\right\}, \\
\tilde{\Theta}_{x}(y) & =\left\{\tilde{\theta}_{x} \mid\left(\tilde{\theta}_{x}, y\right) \in \tilde{\Theta}\right\} .
\end{aligned}
$$

Define probability distribution on $\tilde{\Theta}_{x}$ as follows:

$$
p_{y}\left(\tilde{\theta}_{x}\right)=C \cdot \varphi\left(\left(\tilde{\theta}_{x}, y\right) \mid \hat{\nu}\right) f\left(\tilde{\theta}_{x}, y\right) .
$$

By using $p_{y}\left(\tilde{\theta}_{x}\right)$ and associated statistical moments

$$
\begin{aligned}
m_{x}^{\tilde{\theta}}(y) & =\sum_{\tilde{\theta}_{x} \in \tilde{\Theta}_{x}(y)} \tilde{\theta}_{x} p_{y}\left(\tilde{\theta}_{x}\right), \\
\sigma_{x}^{\tilde{\theta}}(y) & =\sum_{\tilde{\theta}_{x} \in \tilde{\Theta}_{x}(y)}\left(\tilde{\theta}_{x}-m_{x}^{\tilde{\theta}}(y)\right)^{2} p_{y}\left(\tilde{\theta}_{x}\right),
\end{aligned}
$$

we have the following boot-strap scheme for estimating the feature points. First, the variation of $m_{x}^{\bar{\theta}}(y)$ with

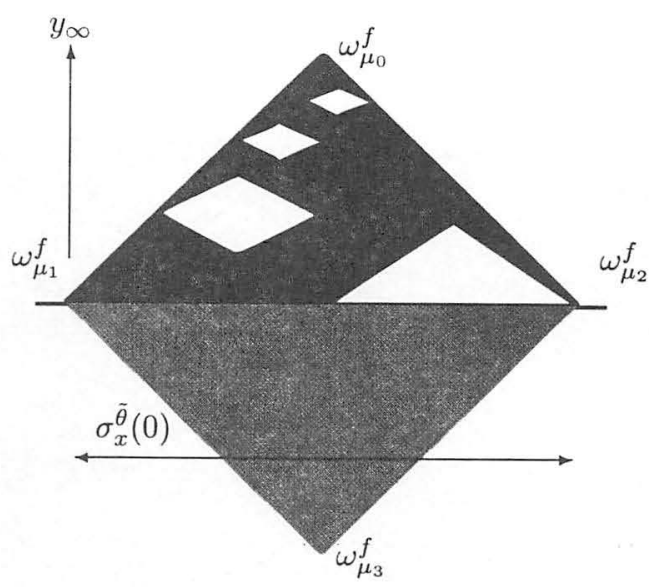

Figure 11: Fractal Representation of Generic Road

respect to $y$ is matched with a linear segment to specify the direction of the vanishing point $\left(x_{\infty}, y_{\infty}\right)$, i.e., $\frac{x_{\infty}-x_{0}}{y_{\infty}}$ :

$$
\begin{gathered}
\sum_{\tilde{\theta}_{x} \in \tilde{\Theta}_{x}(y)}\left|m_{x}^{\tilde{\theta}}(y)-\left(\frac{x_{\infty}-x_{0}}{y_{\infty}} y+x_{0}\right)\right|^{2} \\
\rightarrow \min
\end{gathered}
$$

Next, the distance between $\omega_{\mu_{1}}^{f}$ and $\omega_{\mu_{2}}^{f}$ is evaluated by $\sigma_{x}^{\tilde{\theta}}(0)$ to adjust the vanishing parameter $y_{\infty}$ :

$$
\begin{array}{r}
\sum_{\tilde{\theta}_{x} \in \tilde{\Theta}_{x}(y)}\left|\sigma_{x}^{\tilde{\theta}}(y)-\left(\frac{\sigma_{0}^{\tilde{\theta}}}{y_{\infty}}\right)\left(y_{\infty}-y\right)\right|^{2} \\
\rightarrow \min
\end{array}
$$

Finally, sample set $\tilde{\Theta}_{x}$ is reduced to satisfy

$$
\sum_{\substack{\tilde{\theta}_{x} \in \bar{\Theta}_{x}(y) \\ 0 \leq y \leq y_{\infty}}} p_{y}\left(\tilde{\theta}_{x}\right) \rightarrow \max ,
$$

where $\bar{\Theta}_{x}(y)=\left\{\bar{\theta}_{x}\right\}$ denotes reduction of sample set $\tilde{\Theta}_{x}(y)$ satisfying

$$
m_{x}^{\tilde{\theta}}(y)-2 \sigma_{x}^{\tilde{\theta}}(y) \leq \bar{\theta}_{x} \leq m_{x}^{\tilde{\theta}}(y)+2 \sigma_{x}^{\tilde{\theta}}(y) .
$$

Based on the estimates $x_{0}, x_{\infty}$ and $y_{\infty}$, we can specifies the mapping set as follows:

$$
\begin{aligned}
\hat{\nu} & =\left\{\hat{\mu}_{i}\right\}, \\
\hat{\mu}_{i}(\omega) & =\frac{1}{2}\left[\omega+\omega_{\mu_{i}}^{f}\right] .
\end{aligned}
$$

\section{Experiments}

The representation scheme was tested via experimental studies. Examples of experimental results are shown in 


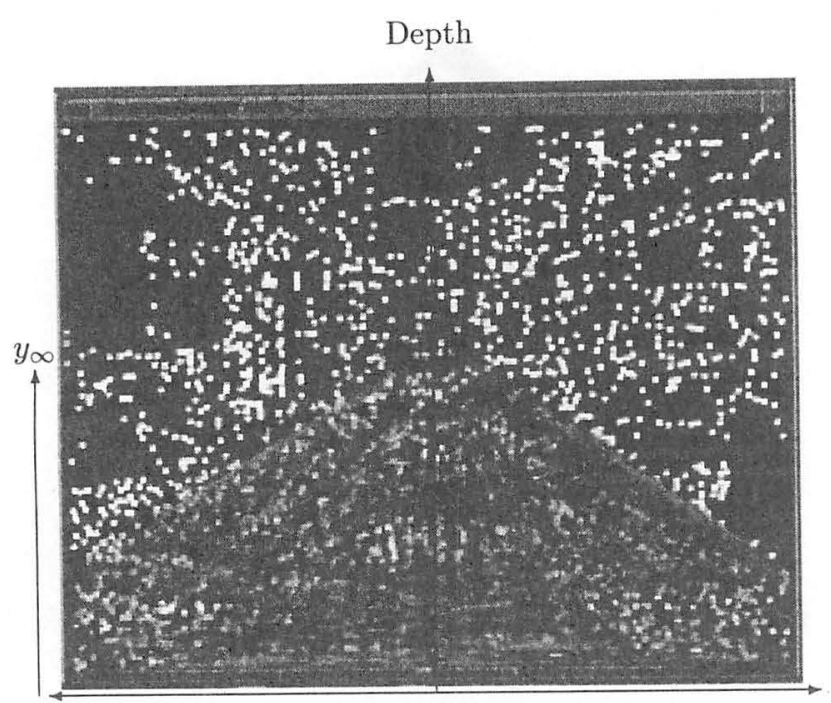

Expansion

Figure 12: Invariant Subset In Stochastic Features

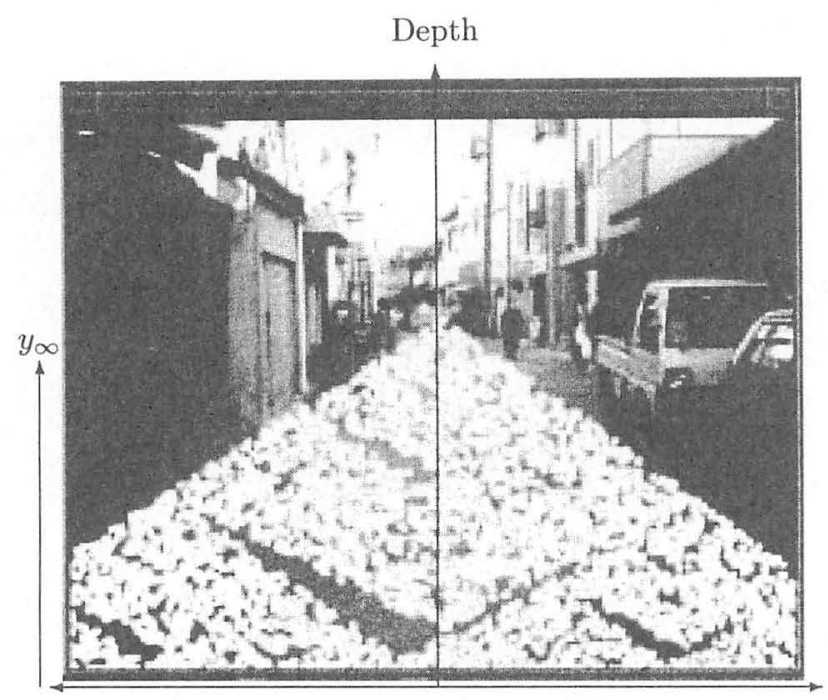

Expansion

Figure 13: Visualized Maneuvering Affordance

Figs. 12 and 13. In figure 12, stochastic features $\tilde{\Theta}$ extracted from the the scene image (Fig. 1) are indicated by white dots. By applying statistical scheme (15), the mapping set (16) was estimated. For estimate $\hat{\nu}$, the finite constraints (6) was tested to detect invariant subset $\Theta \subset \tilde{\Theta}$. Detected invariant structure is visualized by closed links spanning over $\Theta$ as shown in Fig. 12 . The existence of nonempty set $\Theta$, demonstrates that the scene image supports a fractal attractor covering the generic roadway model $\Omega_{\nu}^{f}$. As the results, the vehicle can confine the maneuvering affordance within the fractal attractor generated by $\hat{\nu}$ (Fig. 13).

\section{Concluding Remarks}

Self-similarity and perspective projection were integrated in noisy image to detect the maneuvering affordance embedded in complex scene. By detecting the frequency shift in invariant measure through Laplacian-Gaussian analysis, skewed fractal attractor is estimated in the intentional coordinate system. A generic roadway model is matched with estimated attractor to visualize maneuvering affordance. The representation scheme was verified through experimental studies.

\section{References}

[1] M. F. Barnsley. Fractals Everywhere, Academic Press, 1993.

[2] L. A. Cooper and R. N. Shepard. Chronometric studies of the rotation of mental images. In Visual Information Processing. Academic Press, 1973.

[3] P. W. Coppin, M. Wagner, and J.-L. Li. Collective intelligence via robotic communication: From the view point of robotic network. In Systems, Social and Internationalization Design Aspects of Human-Computer Interaction. Lawrence Erlbaum Associates, Inc., Publishers, 2001.

[4] D. A. Dondis. A Primer of Visual Literacy, MIT Préss, 1973.

[5] M. Ejiri, K. Kamejima, T. Hamada, T. Kimura, and Y. Kimura. Informationssystem für den fahrer eines fahrzeuges. Deutsches Patent DE 69322349 T 2, 1999.

[6] J. J. Gibson. The Ecological Approach to Visual Perception, Houghton Mifflin Company, 1979.

[7] T. Hamada, K. Kamejima, I. Takeuchi, and C. Y. Watanebe. Perceptive display device for displaying and manipulating 2-D or 3-D cursor, 3-D object and associated mark position. United States Patent 4,987,527, 1991.

[8] K. Kamejima. Multi-scale image analysis for stochastic detection of self-similarity in complex texture. In Proc. IEEE-SMC'97, pages 4192-4197, 1997.

[9] K. Kamejima. Fractal representation of image feature associated with maneuvering affordance. In Proc. IEEE-RoMan'02, pages 506-511, 2002.

[10] K. Kamejima. Laplacian-gaussian sub-correlation analysis for the extraction of maneuvering affordance in random image fields. In Proc. IEEE-SEE CSIMTA04, pages 571-576, 2004.

[11] K. Kamejima, R. Dennert, and Y. C. Watanabe. Open logic machine - an interactive perception architecture for unstructured scene analysis -. Advanced Robotics, 8:385-411, 1994.

[12] K. Kamejima, J. Nishihashi, M. Ejiri, M. Yamashita, M. Funabashi, A. Masaki, T. Hamada, I. Nakamura, and K. Matsuzaki. Systems and methods for recycling resources and circulating products. United States Patent 5,436,843, 1995.

[13] K. Kamejima, Y. C. Ogawa, and Y. Nakano. Perception control architecture in image processing system for mobile robot navigation. In Proc. IEEE-IECON'84, pages 52-57, 1984.

[14] Y. Miyashita and H. S. Chang. Neural correlate of pictorial short-term memory in the primate temporal cortex. Nature, 331:568-570, 1988. 